\title{
Comprehensive analysis of inflammatory markers in chronic thromboembolic pulmonary hypertension patients
}

\author{
Diana Zabini', Akos Heinemann², Vasile Foris ${ }^{1}$ Chandran Nagaraj', \\ Patrick Nierlich ${ }^{3}$, Zoltán Bálint ${ }^{1}$, Grazyna Kwapiszewska ${ }^{1,4}$, Irene M. Lang ${ }^{5}$, \\ Walter Klepetko ${ }^{3}$, Horst Olschewski ${ }^{6}$ and Andrea Olschewski ${ }^{1,4}$
}

\begin{abstract}
Affiliations: ${ }^{~ L u d w i g ~ B o l t z m a n n ~ I n s t i t u t e ~ f o r ~ L u n g ~ V a s c u l a r ~ R e s e a r c h, ~ G r a z, ~ A u s t r i a . ~}{ }^{2}$ Institute of Experimental and Clinical Pharmacology, Medical University of Graz, Graz, Austria. ${ }^{3}$ Dept of Surgery, Division of Thoracic Surgery, Medical University of Vienna, Vienna, Austria. "Dept of Anaesthesia and Intensive Care Medicine, Experimental Anaesthesiology, Medical University of Graz, Graz, Austria. ${ }^{5}$ Dept of Internal Medicine II, Cardiology, Medical University of Vienna, Vienna, Austria. ${ }^{6}$ Dept of Internal Medicine, Division of Pulmonology, Medical University of Graz, Graz, Austria.
\end{abstract}

Correspondence: Andrea Olschewski, Ludwig Boltzmann Institute for Lung Vascular Research, Stiftingtalstr. 24, 8010 Graz, Austria.

E-mail: andrea.olschewskidlvr.lbg.ac.at

ABSTRACT Chronic thromboembolic pulmonary hypertension (CTEPH) is associated with chronic inflammation but the pathological mechanisms are largely unknown. Our study aimed to simultaneously profile a broad range of cytokines in the supernatant of pulmonary endarterectomy (PEA) surgical material, as well as prospectively in patients with CTEPH to investigate whether circulating cytokines are associated with haemodynamic and physical characteristics of CTEPH patients.

Herein, we show that PEA specimens revealed a significant upregulation of interleukin (IL)-6, monocyte chemoattractant protein-1, interferon- $\gamma$-induced protein-10 (IP)-10, macrophage inflammatory protein (MIP) $1 \alpha$ and RANTES compared to lung tissue from healthy controls.

In prospectively collected serum, levels of IL-6, IL-8, IP-10, monokine induced by interferon- $\gamma$ (MIG) and MIP1 $\alpha$ were significantly elevated in CTEPH patients compared to age- and sex-matched healthy controls. In serum of idiopathic pulmonary arterial hypertension (IPAH) patients, only IP-10 and MIG were significantly increased. In CTEPH but not in IPAH, IP-10 was negatively correlated with cardiac index, 6-min walking distance and carbon monoxide diffusion capacity. In vitro, IP-10 significantly increased migration of freshly isolated adventitial fibroblasts.

Our study is the first to show that IP-10 secretion is associated with poor pulmonary haemodynamics and physical capacity in CTEPH and might be involved in the pathological mechanism of PEA tissue formation.

@ERSpublications

Increased circulating IP-10 associated with poor pulmonary haemodynamics and physical capacity in CTEPH patients http://ow.ly/yDX7U

For editorial comments see page 842 .

This article has supplementary material available from erj.ersjournals.com

Received: Aug 192013 | Accepted after revision: May 222014 | First published online: July 172014

Support statement: D. Zabini and V. Foris were funded by the Medical University of Graz (PhD programme Molecular Medicine).

Conflict of interest: Disclosures can be found alongside the online version of this article at erj.ersjournals.com

Copyright @ERS 2014 


\section{Introduction}

Chronic thromboembolic pulmonary hypertension (CTEPH) is a disease associated with high morbidity and mortality [1]. CTEPH is caused by obstruction of proximal pulmonary arteries as a consequence of acute and/ or recurrent pulmonary emboli, in situ thrombosis of larger proximal and segmental pulmonary arteries and/ or small vessel pulmonary arteriopathy [2]. The resulting pulmonary hypertension leads to right ventricular dilatation, elevation of end-systolic and end-diastolic pressures, and right heart failure. Although typical risk factors involved in the development of thrombosis, such as mutations in antithrombin, protein S, protein $\mathrm{C}$, factor II or factor V Leiden, were not significantly associated with CTEPH, elevated levels of antiphospholipid antibodies associated with lupus anticoagulant were detected [3]. In addition, the fibrin $\beta$-chain $\mathrm{N}$-terminus from those patients seems to be more resistant to thrombolysis, suggesting an important role for the development of CTEPH but possibly also for pulmonary arterial hypertension (PAH) [4]. Furthermore, a recent study suggests that the differentiation of mesenchymal progenitor cells present in the neointima of the occluded vessels of CTEPH patients might be triggered by factors present in the microenvironment of the included vessel, leading to progressive occlusion [5].

The mechanisms resulting in the persistence and progression of the obstructing fibrothrombotic material in CTEPH requires further elucidation. There is a growing interest in the role of inflammation, and even infection, for the progression of the disease. BONDERMAN et al. [6] have described Staphylococcus infection in CTEPH thrombi. Additionally, they detected that Staphylococcus infection delayed the thrombus resolution in a murine model [6]. In CTEPH, inflammatory cells $\left(\right.$ e.g. $\left.\mathrm{CD} 45^{+}\right)$and collagen-secreting cells have been found to be abundant in the vascular wall $[7,8]$. Furthermore, circulating levels of inflammatory factors such as tumour necrosis factor- $\alpha$, monocyte chemoattractant protein (MCP)-1 and C-reactive protein were shown to be elevated in CTEPH, and MCP-1 showed a positive correlation with pulmonary vascular resistance [9-12].

We have recently reported that CXCL10, also known as interferon- $\gamma$-induced protein-10 (IP-10), causes endothelial dysfunction in primary endothelial cells from human pulmonary arteries [13]. Our working hypothesis was that cytokines like IP-10 mediate the obstructive remodelling of pulmonary arteries in CTEPH. Apart from IP-10, we investigated a broad range of inflammatory factors (interleukin (IL)-8, IL-6, MCP-1, monokine induced by interferon- $\gamma$ (MIG), RANTES, CX3CL1, macrophage inflammatory protein (MIP) $1 \alpha$ and CXCL12) that may be involved in the inflammatory response and may attract cell migration. These factors were investigated in pulmonary endarterectomy (PEA) tissue, PEA supernatant and the serum of newly diagnosed CTEPH and idiopathic pulmonary arterial hypertension (IPAH) patients. We found that in CTEPH patients, IP-10 was negatively correlated with cardiac index, 6-min walking distance (6MWD) and diffusing capacity of the lung for carbon monoxide (DLCO). In addition, IP-10 increased migration of freshly isolated human adventitial fibroblasts in vitro. This suggests an important role of IP-10 in the pulmonary vascular remodelling in CTEPH.

\section{Materials and methods}

Surgical PEA tissue

PEA tissue was obtained from eight CTEPH patients undergoing PEA. The patient characteristics are presented in table 1 . The PEA tissue was transported directly from the operating room to the cell laboratory in endothelial culture media (VascuLife VEGF Cell Culture Medium; LifeLine Technology, Walkersville, MD, USA) and was processed immediately thereafter. For this study, proximal and distal PEA tissue was evaluated. The study protocol for tissue donation was approved by the Institutional Review Board of the Medical University of Vienna (Vienna, Austria) in accordance with national law. Written informed consent was obtained from each individual patient.

In addition, samples from donor lungs, not used for lung transplantation, were used for gene expression studies. This study was also approved by the Institutional Review Board of the Medical University of Vienna in accordance with national law.

\section{Serum samples from CTEPH and IPAH patients and healthy controls}

Blood samples were prospectively taken from a separate cohort of patients who were treated at the Medical University of Graz (Graz, Austria) when undergoing diagnostic or follow-up right heart catheterisation. 16 CTEPH and 11 IPAH patients were enrolled, as well as 16 healthy controls sex- and age-matched with the CTEPH patients and 11 healthy controls sex- and age-matched with the IPAH patients. The samples from the healthy controls $(n=98)$ were prospectively collected by the Biobank of the Medical University of Graz between 2011 and 2013.

All samples underwent the same procedure. Blood samples were left for $30 \mathrm{~min}$ at room temperature and then centrifuged at $1500 \times \mathrm{g}$ for $10 \mathrm{~min}$. Serum was stored in separator tubes (Vacuette premium tubes; 
TABLE 1 Clinical features and haemodynamic parameters

\begin{tabular}{|c|c|c|c|c|c|}
\hline & $\frac{\text { PEA tissue collection }}{\text { CTEPH }}$ & \multicolumn{4}{|c|}{ Serum sample collection } \\
\hline Female/male & $2 / 6$ & $8 / 8$ & $8 / 8$ & $8 / 3$ & $8 / 3$ \\
\hline Age years & $61(44-69)$ & $64(34-86)$ & $63(34-86)$ & $65(30-83)$ & $63(34-86)$ \\
\hline Heart rate bpm & 95 (92-98) & 77.5 (65.5-87.5) & ND & $78(67-80)$ & ND \\
\hline PVR dyn $\cdot s \cdot m^{-5}$ & $851(733-1194)$ & $788(499-993)$ & ND & $550(462-722)$ & ND \\
\hline mPAP mmHg & $54(48-57)$ & $45.5(40-59)$ & ND & $40(35.5-46)$ & ND \\
\hline Cardiac output L·min ${ }^{-1}$ & $4.5(3.6-4.7)$ & $3.8(3.5-5.1)$ & ND & $5.3(4.6-5.7)$ & ND \\
\hline Cardiac index $\mathrm{L} \cdot \mathrm{min}^{-1} \cdot \mathrm{m}^{-2}$ & $2.2(2-2.2)$ & $2.1(1.9-2.5)$ & ND & $2.8(2.1-3.1)$ & ND \\
\hline Right atrial pressure $\mathrm{mmHg}$ & ND & $10(5.8-13)$ & ND & $7(5-10)$ & ND \\
\hline 6MWD m & 208 (185.5-241.5) & $350(248-428)$ & ND & $408(327.8-441)$ & ND \\
\hline \multicolumn{6}{|l|}{ NYHA } \\
\hline I & 0 & 1 & ND & 0 & ND \\
\hline II & 0 & 6 & ND & 4 & ND \\
\hline III & 8 & 9 & ND & 7 & ND \\
\hline IV & 0 & 0 & ND & 0 & ND \\
\hline
\end{tabular}

Data are presented as $\mathrm{n}$ or median (interquartile range), except for age which is presented as median (range). PEA: pulmonary endarterectomy; CTEPH: chronic thromboembolic pulmonary hypertension; IPAH: idiopathic pulmonary arterial hypertension; PAWP: pulmonary artery wedge pressure; $\mathrm{PCO}_{2}$ : partial pressure of carbon dioxide; PVR: pulmonary vascular resistance; mPAP: mean pulmonary artery pressure; 6MWD: 6-min walking distance; CRP: C-reactive protein; NT-proBNP: N-terminal pro-brain natriuretic peptide; NYHA: New York Heart Association; ND: not defined.

Greiner Bio-One $\mathrm{GmbH}$, Kremsmünster, Austria). All samples were aliquoted and stored at $-80^{\circ} \mathrm{C}$ in the Biobank of the Medical University of Graz. Written informed consent was obtained from all subjects. The study was approved by the Institutional Review Board of the Medical University of Graz in accordance with national law.

Isolation of adventitial fibroblasts from donor lungs

Adventitial fibroblasts were isolated from pulmonary arteries from non-transplantable donor lungs by removing the adventitial layer. The adventitial fibroblast tissue was cut and the fragments were resuspended in adventitial fibroblast medium (FibroLife Medium, LS-1038 supplements; LifeLine) and cultured in T75 culture flasks.

\section{Immunohistochemistry}

Fresh surgical PEA material and human donor lungs were fixed in $4 \%$ formaldehyde for $24 \mathrm{~h}$ and embedded in paraffin blocks. The sliced paraffin tissue (2- $\mu$ m thick) was placed on Capillary Gap Microscope Slides (Dako REAL; Dako, Ely, UK) and kept for further use at room temperature. The immunohistochemical stainings for IP-10 (1:250, ab9807; Abcam, Cambridge, UK), IL-8 (1:100; ab7747; Abcam), MCP-1 (1:100, ab73680; Abcam) and IL-6 (1:100, ab6672; Abcam) were performed with the 3,3'-diaminobenzidine peroxidase substrate kit from R\&D Systems (Minneapolis, MN, USA). For the haematoxylin-eosin staining, the protocol of Gill was used. The staining was analysed by an experienced pathologist who was blinded to the patient characteristics. For the collagen fibre staining, the Masson's Trichrome staining protocol was used according to the manufacturer's instructions. The stainings for vimentin (1:100, ab11256; Abcam), $\alpha$ actin (1:500; Everest Biotech Ltd, Upper Heyford, UK), fibronectin (1:250, ab23750; Abcam), CD45 (1:200, ab10558; Abcam), CD68 (1:250, sc-20060; Santa-Cruz Biotechnology Inc., Dallas, TX, USA), MIP1 $\alpha$ (1:50, PA5-32496; Thermo Scientific, Rockford, IL, USA) and CXCL12 (1:100, sc-28876; Santa-Cruz Biotechnology Inc.) were performed with the Vektor ImmPRESS reagent kit (Vector Laboratories Ltd, Peterborough, UK). 


\section{Determination of the supernatant of PEA tissue}

After removal, the PEA samples from the eight CTEPH patients were immediately put into endothelial cell basal medium supplemented with 5\% fetal calf serum (FCS) and antibiotics (VascuLife VEGF Cell Culture Medium; LifeLine Technology) [14]. The tissue was cut into small pieces and incubated for $18 \mathrm{~h}$ at $37^{\circ} \mathrm{C}$ in DMEM-F12 medium (Gibco) with 0.3\% FCS, glutamine, penicillin and streptomycin (1\% each).

A similar procedure was performed for supernatant collection from primary human cells from donor lungs (pulmonary arterial endothelial cells, smooth muscle cells and fibroblasts). The supernatant was collected and stored at $-80^{\circ} \mathrm{C}$.

\section{Measurement of cytokines}

IP-10, IL-8, RANTES, MIG and MCP-1 were measured with the Human Chemokine Kit Cytometric Bead Array (Becton Dickinson, Franklin Lakes, NJ, USA), whereas IL-6, fractalkine and MIP1 $\alpha$ contents in the supernatant and serum were determined using the IL-6, fractalkine and MIP1 $\alpha$ Flex sets (558276; 560265; 558325; Becton Dickinson). The measurements were performed on a FACS Canto II flow cytometer (Becton Dickinson). CXCL12 was measured in the supernatant and serum via ELISA (human CXCL12/SDF1 $\alpha$ Quantikine ELISA kit; R\&D Systems).

\section{RNA isolation, c-DNA synthesis and real-time PCR}

The trizol protocol was applied for total RNA isolation from PEA material of eight patients and samples from control healthy donor lungs not used for lung transplantation. A Nanodrop 2000c spectrophotometer (PeQlab, Sarisbury Green, UK) was used to quantify the concentration and the purity of the isolated total RNA. The total RNA was converted to cDNA using a RevertAid H Minus First Strand cDNA Synthesis kit (Fermentas; Thermo Fischer Scientific, Schwerte, Germany). Real-time PCR analysis was performed by a Lightcycler 480 (Roche Diagnostic International AG, Rotkreuz, Switzerland). As a housekeeping gene human B2M was used. Primers used are listed in table S6.

\section{Migration assay}

Adventitial fibroblasts were serum-starved over-night in basal medium (FibroLife Medium; LifeLine Cell Technology, Frederick, MD, USA). Sub-confluent cells were harvested with trypsin-EDTA and $8.0 \times 10^{5}$ cells were placed in cell culture inserts (PIEP12R48; Merck Millipore, Darmstadt, Germany). The bottom of the wells were filled with $2 \mu \mathrm{g} \cdot \mathrm{mL}^{-1}$ isotype control or isotype control with IP-10 $\left(10 \mathrm{ng} \cdot \mathrm{mL}^{-1}\right)$. Additional wells were filled with IP-10 $\left(10 \mathrm{ng} \cdot \mathrm{mL}^{-1}\right)$ with CXCR3 blocking antibody $\left(2 \mu \mathrm{g} \cdot \mathrm{mL}^{-1}\right)$ or IP-10 neutralising antibody $\left(2 \mu \mathrm{g} \cdot \mathrm{mL}^{-1}\right)$. After $6 \mathrm{~h}$ the cells were fixed with methanol and stained with haematoxylin. 20 pictures of five random regions for each filter were made with a light microscope (20X LCPlanFl objective; Olympus Optical Company LTD, Tokyo, Japan). The number of cells that migrated to the bottom of the filters was counted.

\section{Proliferation assay}

To investigate the effect of IP-10 on human adventitial fibroblast proliferation the following protocol was applied: 10000 cells (from three different donors) were seeded in 96-well plates. Prior to stimulation adventitial fibroblasts were kept in quiescent medium (VascuLife basal medium with 1\% penicillin/ streptomycin and $0.1 \%$ FCS; LifeLine Technology) for $24 \mathrm{~h}$. Cells were then treated for $24 \mathrm{~h}$ with 1,10 and $100 \mathrm{ng} \cdot \mathrm{mL}^{-1} \mathrm{IP}-10$ or were kept under control conditions. The proliferation of adventitial fibroblasts was determined by $\left[{ }^{3} \mathrm{H}\right]$-thymidine (Biotrend Chemikalien $\mathrm{GmbH}$, Cologne, Germany) incorporation as an index of DNA synthesis and measured as radioactivity by a scintillation counter (Wallac 1450 MicroBeta TriLux Liquid Scintillation Counter and Luminometer; PerkinElmer Life and Analytical Sciences, Turku, Finland). Experiments were performed in quintuplicate and repeated four to seven times.

\section{Statistical analysis}

Data are presented as median (interquartile range (IQR)), unless otherwise stated. Intergroup differences were assessed by factorial analysis of variance with post hoc Bonferroni test or Mann-Whitney U-test as appropriate. The Spearman test was performed for intergroup correlations. A p-value $<0.05$ was considered significant.

\section{Results}

Inflammatory factors secreted by PEA tissue

The clinical features of the patients with CTEPH are reported in table 1. Cytokines released by the resident cells of proximal and distal PEA tissue from eight patients were measured in the supernatant. IL-8, IL-6, MCP-1, IP-10, CXCL12, MIP1 $\alpha$, RANTES and MIG were present at high concentrations as compared to 
CX3CL1/fractalkine (fig. 1). Low levels of cytokines were detected in the supernatant of primary human pulmonary arterial endothelial, smooth muscle cells and fibroblast (table S1). In the PEA material itself, mRNA levels were determined. IP-10, IL-6, MCP-1, MIP1 $\alpha$ and RANTES mRNA were significantly upregulated compared to healthy human lung tissue (fig. 2a-i). Immunohistochemical staining of the PEA tissue showed expression of IP-10, IL-8, IL-6, MCP-1, CXCL12, and MIP1 $\alpha$ (fig. 2j-O). Stainings for the same factors were also performed in donor lungs (fig. S1). The receptors for all these factors were also present in the PEA tissue (fig. S2).

\section{Circulating inflammatory cytokines in CTEPH and IPAH patients}

We prospectively enrolled CTEPH and IPAH patients and sex- and age-matched healthy controls to investigate the circulating inflammatory cytokines. Blood sampling was performed during right heart catheterisation. The clinical features and haemodynamic parameters of the patients and the controls are shown in table 1 . Flow cytometry and ELISA assay performed on the serum samples showed a significantly elevated level of IL-8, IL-6, IP-10, MIG and MIP1 $\alpha$ in the serum of CTEPH patients as compared to healthy controls (fig. 3 and table S2), while serum levels of RANTES were significantly decreased. To elucidate if the observed cytokine profile is specific for CTEPH, we assessed the same inflammatory cytokines in the serum of IPAH patients and their age- and sex-matched controls. The demographics and pulmonary haemodynamics of the IPAH patients are listed in table 1. In IPAH, IP-10 and MIG but not IL-6, IL-8 and MIP1 $\alpha$ were significantly elevated (fig. 4).

\section{IP-10 and IL-6 are significantly correlated with haemodynamics in CTEPH}

Next we performed Spearman analysis between the significantly elevated cytokine levels and haemodynamics. The detailed analysis is shown in table S3. In CTEPH, there was a significant negative correlation between IP-10 and cardiac output $(\rho=-0.71)$ and cardiac index $(\rho=-0.54)$ (fig. 5). In addition, there was a significant positive correlation between IL- 6 and pulmonary vascular resistance $(\rho=0.52)$, right atrial pressure $(\rho=0.62)$ and $\mathrm{N}$-terminal pro-brain natriuretic peptide (NT-proBNP; $\rho=0.51$ ) (fig. 5). None of the other inflammatory cytokines demonstrated any significant correlations with haemodynamic parameters. In addition, IP-10 serum levels were negatively correlated with DLCO $(\rho=-0.57$ for single breath DLCO and $\rho=-0.62$ for DLCO/alveolar volume).

In the IPAH patients, IL-6 but not IP-10 was significantly correlated with haemodynamic parameters (tables S4 and S5). IL-6 was significantly negatively correlated with cardiac output $(\rho=-0.63)$ and cardiac index $(\rho=-0.86)$. There was a significant positive correlation with New York Heart Association functional class $(\rho=0.66)$, C-reactive protein $(\rho=0.67)$, uric acid $(\rho=0.71)$ and NT-proBNP $(\rho=0.85)$. Taken together, these results indicate that IL-6 is strongly associated with prognostic markers in IPAH, but that IP10 is only associated with haemodynamics in CTEPH.

\section{Correlations of cytokine levels with 6MWD}

In CTEPH patients, only IP-10 and IL-6 were significantly correlated with exercise capacity, as assessed by 6 MWD ( $\rho=-0.66$ and $\rho=-0.64$, respectively) (fig. 5 and table S3).

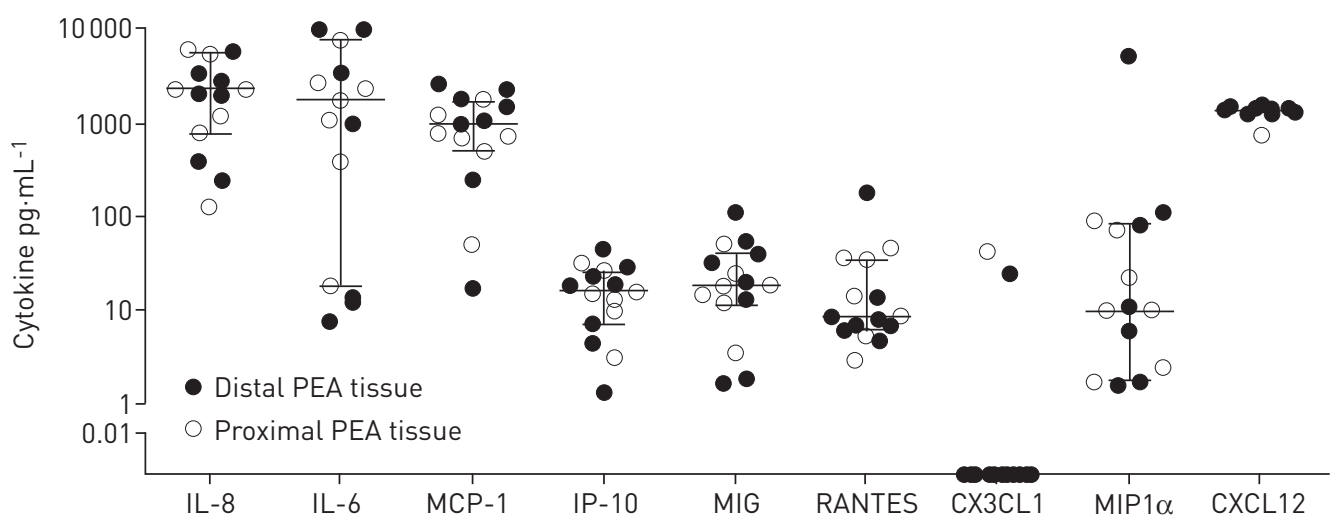

FIGURE 1 Cytokines in the supernatant of pulmonary endarterectomy (PEA) tissue. Results of flow cytometry and ELISA measurements in the supernatant of PEA tissue obtained from eight different patients. Data are presented as median (interquartile range). Each data point is a mean of ELISA/fluorescence-activated cell sorter measurements obtained from three to 12 independent PEA materials. IL: interleukin; MCP: monocyte chemoattractant protein; IP: interferon- $\gamma$ induced protein; MIG: monokine induced by interferon- $\gamma$; MIP: macrophage inflammatory protein. 

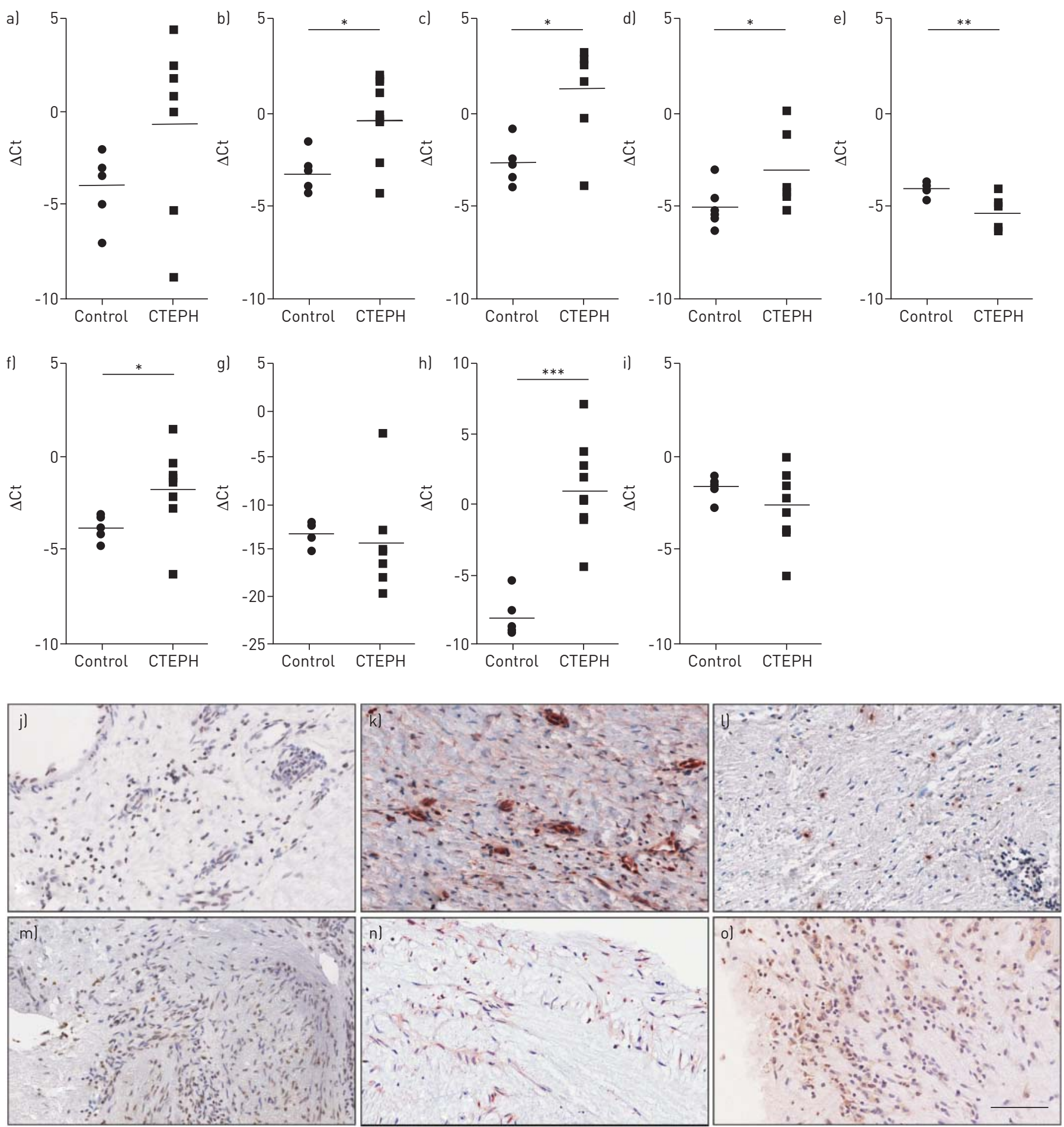

FIGURE 2 Cytokines in pulmonary endarterectomy (PEA) tissue. Gene upregulation of a) interleukin (IL)-8, b) IL-6, c) monocyte chemoattractant protein (MCP)-1, d) interferon- $\gamma$-induced protein (IP)-10, e) monokine induced by interferon- $\gamma$, f) RANTES, g) CX3CL1, h) macrophage inflammatory protein (MIP) $1 \alpha$ and i) CXCL12 in PEA tissue compared to control lung. ${ }^{*}: p<0.05{ }^{* *}$ : $p<0.01{ }^{* * *}$ : $p<0.001$. Immunohistochemical staining of j) IL-8, k) IL-6, l) MCP-1, m) IP-10, n) MIP1 $\alpha$ and o) CXCL12. CTEPH: chronic thromboembolic pulmonary hypertension. Scale bar $=100 \mu \mathrm{m}$.

\section{Infiltration of the PEA material by fibroblasts and inflammatory cells}

Fixed PEA tissue in serial sectional analysis from Trichrome staining showed an accumulation of collagen (blue) and cell infiltration (red) (fig. 6). Immunohistochemistry showed that most of the infiltrating cells were positively stained for $\alpha$-actin, fibronectin and vimentin in the PEA tissue, especially in the distal part of the removed tissue. In addition, inflammatory cells $\left(\mathrm{CD} 45^{+}\right)$were also detected, predominantly mononuclear cells 

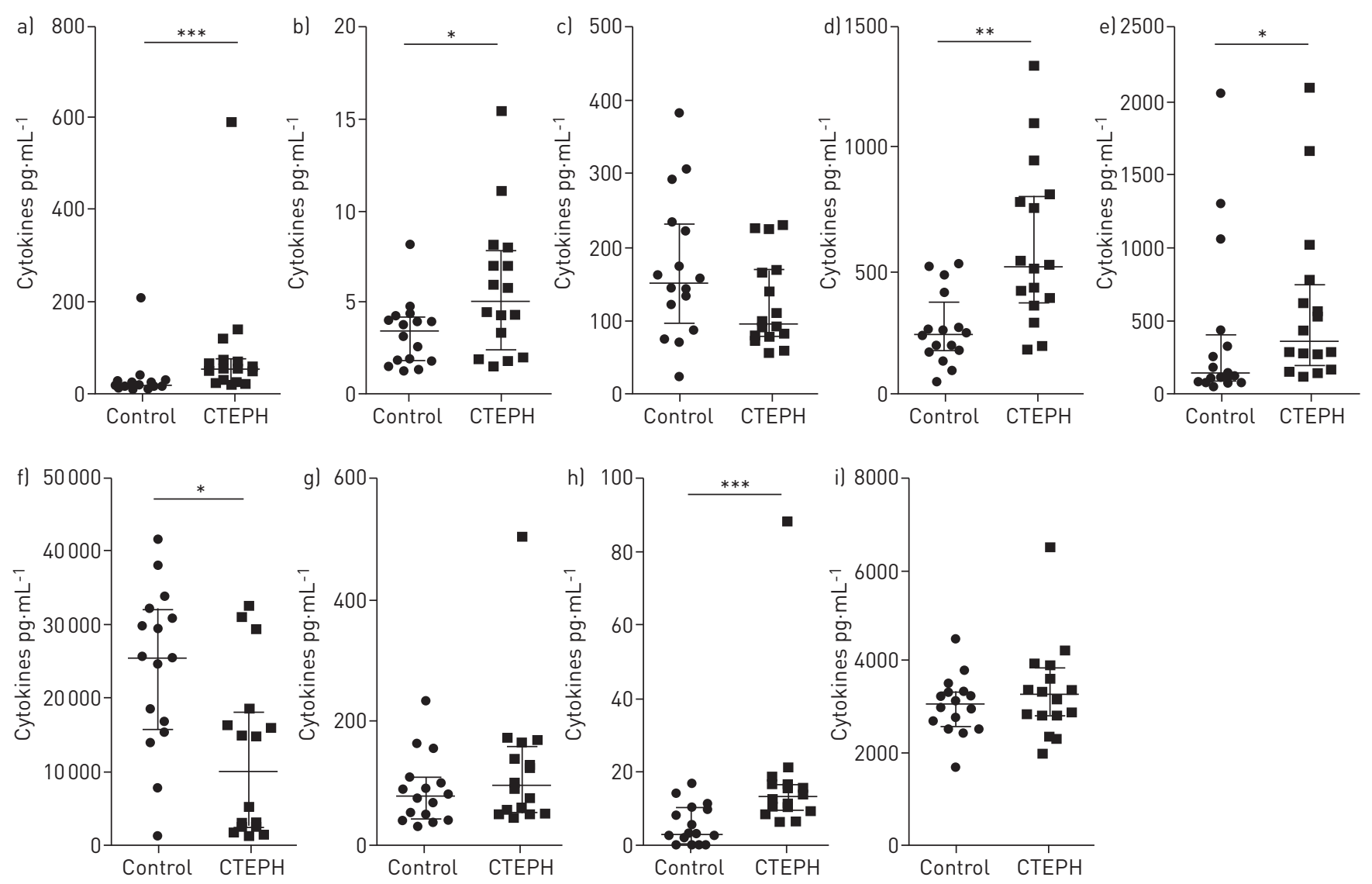

FIGURE 3 Cytokines in the serum of chronic thromboembolic pulmonary hypertension (CTEPH) patients versus matched controls. a) Interleukin (IL)-8, b) IL-6, c) monocyte chemoattractant protein-1, d) interferon- $\gamma$-induced protein-10, e) monokine induced by interferon- $\gamma$, f) RANTES, g) CX3CL1, h) macrophage inflammatory protein-1 $\alpha$ and i) CXCL12. Serum samples were taken from venous blood during right heart catheterisation in CTEPH patients and compared to matched controls. ${ }^{*}: \mathrm{p}<0.05 ;{ }^{* *}: \mathrm{p}<0.01 ;{ }^{* * *}: \mathrm{p}<0.001$.

consistent with lymphocytes, with a minority of macrophages and neutrophils (fig. 6 and fig. S3). Collagen $\alpha$ actin, fibronectin, vimentin and CD45 were also shown in human donor lungs (fig. S4).

\section{IP-10 induces adventitial fibroblast migration}

In transwell migration assays, we found that IP-10 significantly attracted human pulmonary arterial adventitial fibroblasts. The effect of IP-10 was diminished by co-treatment with an CXCR3 blocking antibody or an IP-10 neutralising antibody (fig. 7a). In contrast, IP-10 did not alter either the proliferative behaviour of the adventitial fibroblast or lead to increased activation of F-actin even with the highest used dosage (fig. $7 \mathrm{~b}$ and c).

\section{Discussion}

This study is the first to simultaneously profile a broad range of cytokines in PEA surgical material, its supernatant and in an independent cohort of patients with CTEPH. In addition, we compared the circulating cytokine levels obtained from CTEPH patients with IPAH patients and control subjects. Our results indicate a significant dysregulation of circulating inflammatory cytokines in both CTEPH and IPAH patients. Among IPAH patients, factors indicating decompensation of the right ventricle, such as $6 \mathrm{MWD}$, uric acid, NT-proBNP, etc. were strongly associated with IL-6 levels. This agrees with previous findings from SOON et al. [15] and HUMBERT et al. [16]. We found that in CTEPH patients such associations were also present with IP-10 levels. IP-10 may be of particular importance because it induces fibroblast migration, a factor that may contribute to the high cellularity of the distal PEA material with collagen producing cells, as observed by OGAWA et al. [17]. We demonstrated that the dysregulation of serum cytokines in CTEPH does not only include IP-10, IL-6 and IL-8, but also MIG and MIP1 $\alpha$. Correspondingly, in PEA tissue we detected significantly increased levels of IL-6, MCP-1, IP-10, RANTES and MIP1 $\alpha$ and we showed that the proteins are present in the PEA tissue. 

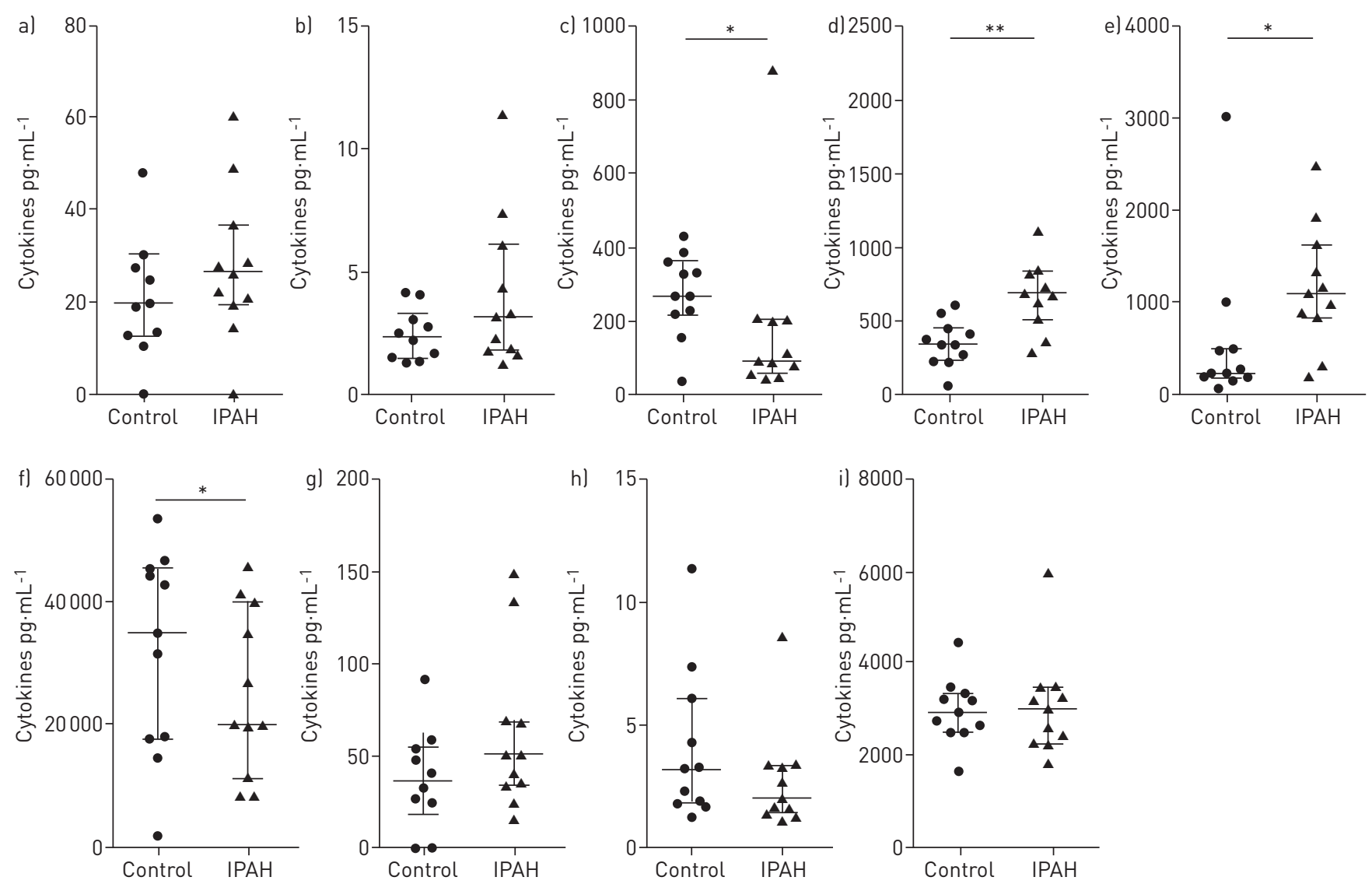

FIGURE 4 Cytokines in serum of idiopathic pulmonary arterial hypertension (IPAH) patients compared to matched controls. a) Interleukin (IL)-8, b) IL-6, c) monocyte chemoattractant protein-1, d) interferon- $\gamma$-induced protein-10, e) monokine induced by interferon- $\gamma$, f) RANTES, g) CX3CL1, h) macrophage inflammatory protein- $1 \alpha$ and i) CXCL12. Samples were taken from venous blood during right heart catheterisation. ${ }^{*}: \mathrm{p}<0.05 ;{ }^{* *}: \mathrm{p}<0.01$.

CTEPH is a rare and late consequence of venous thromboembolism and it is associated with prominent obstructions in larger pulmonary arteries [18]. Despite some evidence of dysregulated thrombolysis, the pathological mechanisms of CTEPH remain poorly understood and may involve inflammation, in situ thrombosis and endothelial dysfunction. A deleterious effect of chronic inflammation in CTEPH patients is suggested by studies showing that inflammation not only contributes to the pathogenesis of CTEPH, but also to the development of the disease [19]. An increased prevalence of chronic inflammatory diseases was found in CTEPH patients [20] and the presence of Staphylococcal antigen in most patients with ventriculoatrial shunt or pacemaker-related CTEPH [6]. Another retrospective study reported that the inflammatory marker C-reactive protein was significantly elevated in patients with CTEPH [12]. In a follow-up study, based on their in vitro data, WYNANTS et al. [21] postulated that C-reactive protein may contribute to the persistence of obstructing material in pulmonary arteries in CTEPH.

Pro-inflammatory cytokine activation, including IL-6, has been described in patients with IPAH $[15,16]$, and recently in patients with isolated right ventricular dysfunction due to CTEPH [22]. In addition, haemodynamic instability after PEA has been associated with higher post-operative plasma concentrations of IL-6 and IL-8 [15], suggesting that these cytokines may act as biomarkers to identify patients at risk for developing residual pulmonary hypertension after PEA surgery. Our study confirms the finding of increased levels of IL-6 in the CTEPH serum and extends it by a significant correlation with markers of right ventricular function. In addition, it shows that IL-8 is not only elevated in post-operative but also in preoperative patients. In both the IPAH and CTEPH cohorts, IL-6 was significantly correlated with haemodynamics, although the average level was not significantly increased in IPAH compared to matched controls.

Previous studies investigating the role of chemokines in pulmonary hypertension have primarily focused on PAH. In lung homogenate of IPAH patients (formerly precapillary pulmonary hypertension), increased mRNA levels of the CC chemokine MlP1 $\alpha$ [23], and subsequently RANTES, was reported [24]. We detected significantly increased MlP1 $\alpha$ and RANTES in the PEA tissue; however, in the serum of CTEPH patients, 

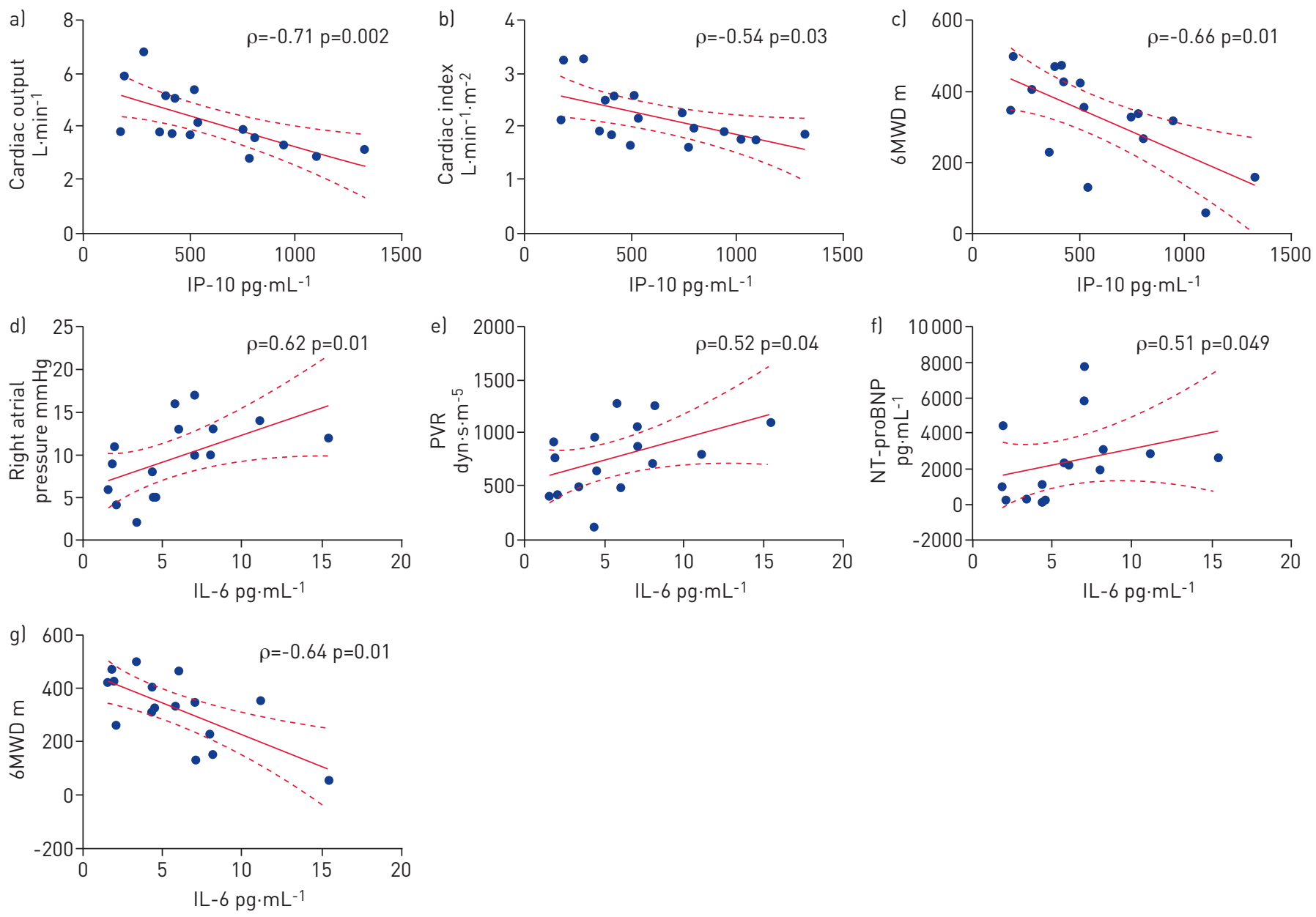

FIGURE 5 Correlation of the serum levels of interferon- $\gamma$-induced protein (IP)-10 and interleukin (IL)- 6 with haemodynamic and physical parameters in chronic thromboembolic pulmonary hypertension (CTEPH) patients. IP-10 correlation with a) cardiac output, b) cardiac index and c) 6-min walking distance (6MWD). IL-6 correlation with d) right atrial pressure, e) pulmonary vascular resistance (PVR), f) N-terminal pro-brain natriuretic peptide (NT-proBNP) and g) 6MWD. —: Spearman correlation coefficient $(\rho) ;-\ldots$ - - $95 \%$ CI of the fit.

only MlP1 $\alpha$ was elevated and RANTES was decreased. KIMURA et al. [11] reported a correlation of elevated pulmonary vascular resistance with expression of the CCL2 chemokine MCP-1 in the plasma and large pulmonary arteries of CTEPH patients. However, they did not compare their findings in the CTEPH population with healthy controls. Our study included sex- and age-matched healthy controls for both the CTEPH and IPAH patients. This showed no significant change of MCP-1 serum level. In our CTEPH population, MIG was significantly elevated as compared with healthy controls. Finally, in line with previous observations in PAH [25], we found that CXCL10/IP-10 was significantly elevated in CTEPH patients. This chemokine is secreted by the cells in the PEA tissue and may attract fibroblasts into the thromboembolic material. Therefore, it could be a link between the persistence of the obstructing fibrothrombotic material and the haemodynamic deterioration.

A major strength of our study is the comprehensive correlation analysis of circulating cytokines with haemodynamics, gas exchange and physical parameters of CTEPH patients. IP-10 was increased both on the mRNA and protein level in PEA tissue, as well as in the serum of CTEPH patients. We observed a significant negative correlation of IP-10 with classic indexes of right heart function (cardiac output and cardiac index), as well as 6MWD. The consistent upregulation of IP-10 in the PEA tissue together with its effect as an inducer of fibroblasts migration and the correlation with haemodynamics points to an important pathological function in the development of CTEPH.

This study was exploratory and included a relatively low amount of PEA material. We employed multiple testing on the circulating levels of cytokines and patients haemodynamics with numerous correlation analyses and comparisons between small numbers of patients and their matched controls. However, we 

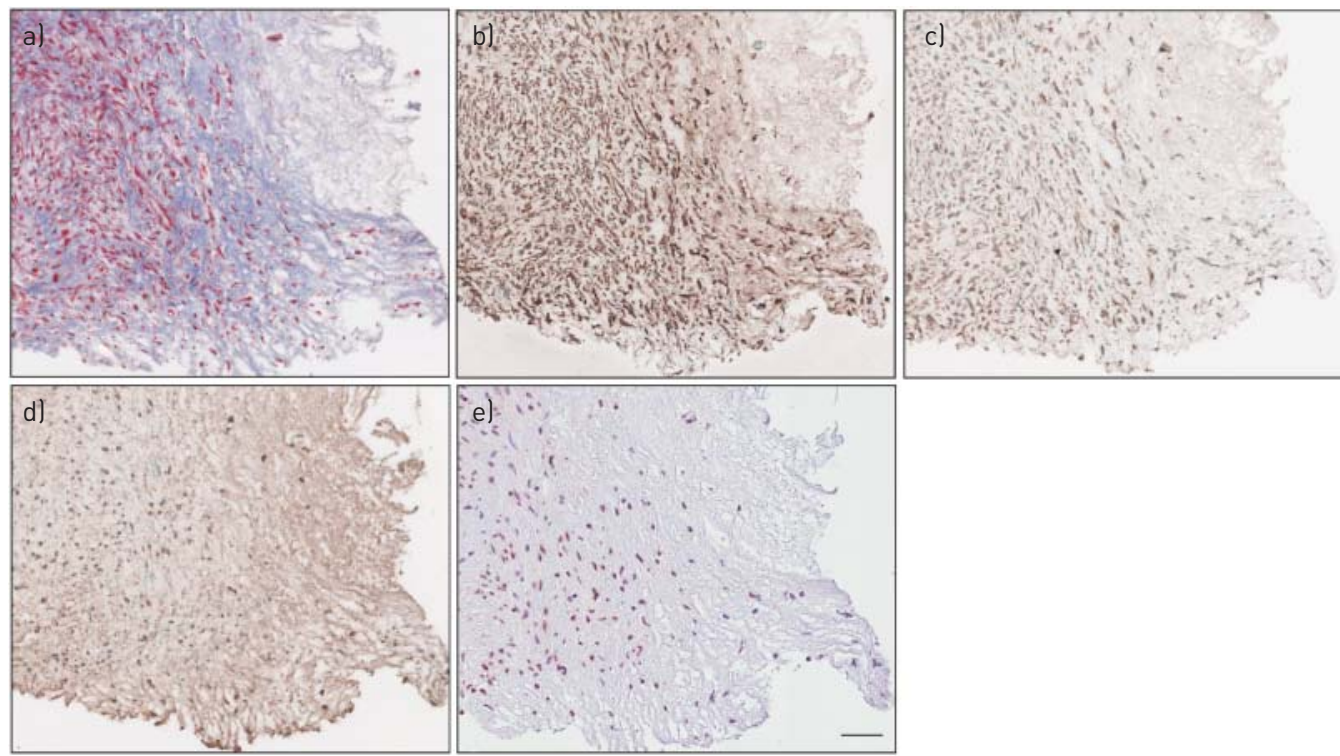

FIGURE 6 Representative photomicrographs of serial sections of pulmonary endarterectomy tissue. Sections were stained with a) trichrome to show collagen, or immunostained for b) vimentin, c) $\alpha$-smooth muscle actin, d) fibronectin and e) CD45. All images are representative of eight patients. Scale bar $=50 \mu \mathrm{m}$.

included clinically well-characterised CTEPH and IPAH patients and sex- and age-matched healthy controls that were independently and prospectively enrolled in the Biobank of the Medical University of Graz. To confirm the diagnostic value of the inflammatory markers, such investigations should be extended to a larger number of patients in a prospective study.
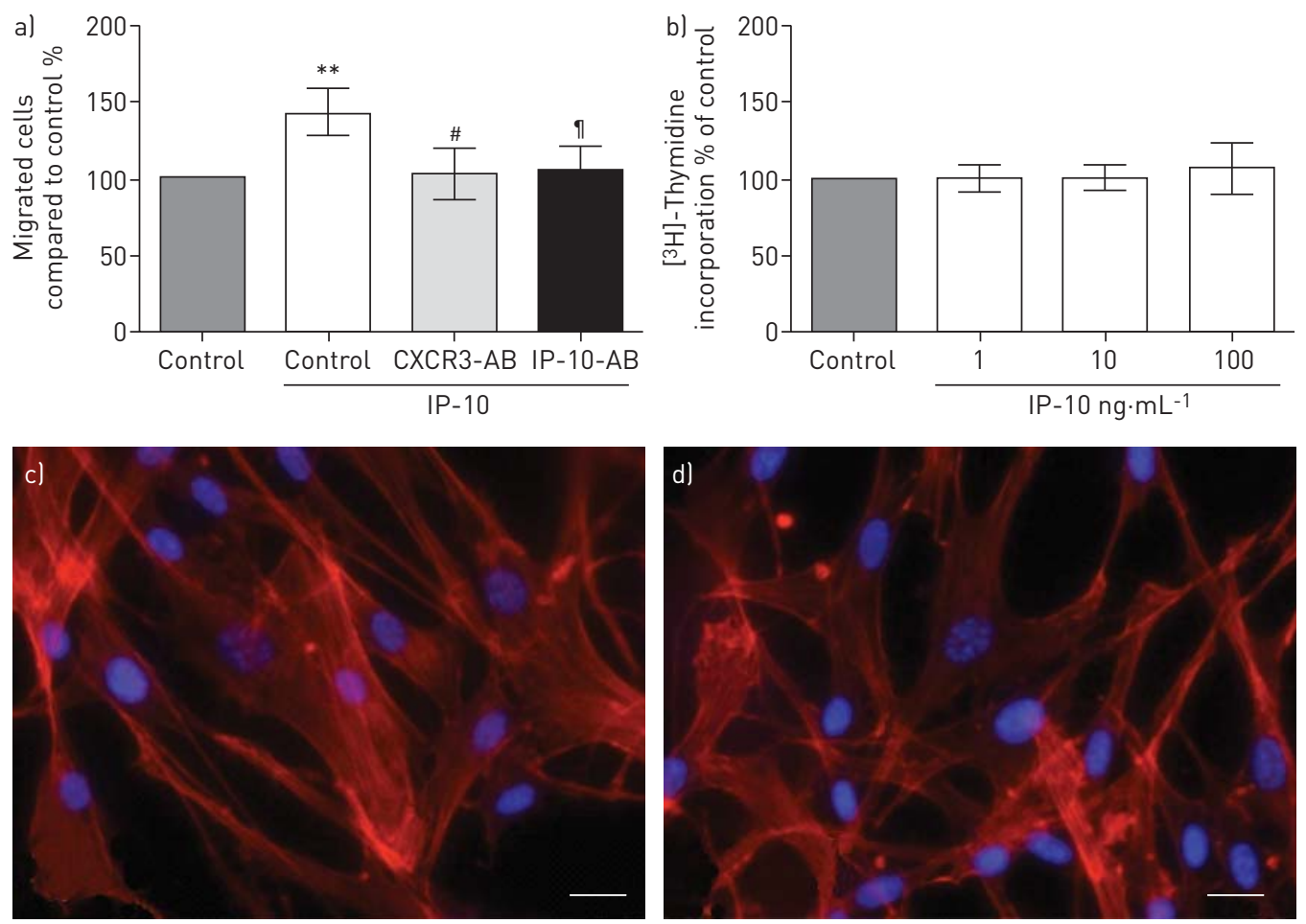

FIGURE 7 Interferon- $\gamma$-induced protein (IP)-10 causes adventitial fibroblast migration. a) Migrated adventitial fibroblasts towards IP-10 stimuli compared to untreated control, or treatment with CXCR3-antibody (AB) or with IP-10 neutralising $A B .{ }^{* *}: \mathrm{p}<0.01{ }^{*}: \mathrm{p}<0.01,{ }^{\uparrow}: \mathrm{p}<0.05$ compared to IP-10 treated control. b) Proliferating adventitial fibroblasts on 1, 10 and $100 \mathrm{ng} \cdot \mathrm{mL}^{-1} \mathrm{IP}-10$ stimuli compared to unstimulated control. Adventitial fibroblasts stimulated for $24 \mathrm{~h} \mathrm{c}$ ) without or d) with IP-10 were stained for F-actin. Scale bar $=20 \mu \mathrm{m}$. 
We can only speculate on the mechanisms underlying progressive obstruction of the pulmonary arteries in CTEPH. However, the importance of IP-10 for endothelial dysfunction in the pulmonary circulation has been recently shown [13]. In the current study we showed that IP-10 specifically boosts adventitial fibroblast migration. Thus, local IP-10 may attract fibroblasts from the adventitia or perhaps circulating fibrocytes into the thromboembolic material leading to a persistent accumulation of those cells that secrete collagen and may cause growth and stiffening of this endovascular material. Also MCP-1, MlP1 $\alpha$ and IL- 6 were shown to promote migration of monocytes, macrophages and fibroblasts [26, 27]. Furthermore, MCP-1induced inflammation can lead to neointimal hyperplasia $[28,29]$ and also stimulates fibroblast proliferation and collagen secretion [30]. Finally, in line with our observations, inflammatory cell infiltration was observed in the distal vascular end of the PEA material [8]. These findings constitute further evidence that cellular infiltration and proliferation as well as collagen production are among the factors that lead to progressive occlusion of the pulmonary arteries in CTEPH and that IP-10 may play a central role.

In conclusion, we demonstrate that IP-10 is elevated on the mRNA and protein level of PEA tissue, as well as in the serum of CTEPH patients. The pathophysiological relevance of this upregulation is supported by the fact that circulating IP-10 levels are correlated with poor haemodynamic and physical parameters. In addition, IP10 facilitates fibroblast migration, which might contribute to the progression of the disease. Further studies to define the temporal changes of IP-10 during disease progression and treatment are warranted.

\section{Acknowledgements}

We would like to thank Elvira Stacher (Institute of Pathology, Medical University of Graz, Graz, Austria) for her help in the analysis of the PEA tissue stainings, and Alexander Avian (Institute for Medical Informatics, Statistics and Documentation, Medical University of Graz) for analysis of the data, as well as Martina Ofner (Institute of Experimental and Clinical Pharmacology, Medical University of Graz) and Sabine Halsegger (Dept of Anaesthesia and Intensive Care Medicine, Experimental Anaesthesiology, Medical University of Graz) for their excellent technical support.

\section{References}

1 Fedullo PF, Auger WR, Kerr KM, et al. Chronic thromboembolic pulmonary hypertension. N Engl J Med 2001; 345: $1465-1472$.

2 Sacks RS, Remillard CV, Agange N, et al. Molecular biology of chronic thromboembolic pulmonary hypertension. Semin Thorac Cardiovasc Surg 2006; 18: 265-276.

3 Wolf M, Boyer-Neumann C, Parent F, et al. Thrombotic risk factors in pulmonary hypertension. Eur Respir J 2000; 15: 395-399.

4 Miniati M, Fiorillo C, Becatti M, et al. Fibrin resistance to lysis in patients with pulmonary hypertension other than thromboembolic. Am J Respir Crit Care Med 2010; 181: 992-996.

5 Firth AL, Yao W, Ogawa A, et al. Multipotent mesenchymal progenitor cells are present in endarterectomized tissues from patients with chronic thromboembolic pulmonary hypertension. Am J Physiol Cell Physiol 2010; 298: C1217-C1225.

6 Bonderman D, Jakowitsch J, Redwan B, et al. Role for staphylococci in misguided thrombus resolution of chronic thromboembolic pulmonary hypertension. Arterioscler Thromb Vasc Biol 2008; 28: 678-684.

7 Yi ES, Kim $\mathrm{H}, \mathrm{Ahn} \mathrm{H}$, et al. Distribution of obstructive intimal lesions and their cellular phenotypes in chronic pulmonary hypertension. A morphometric and immunohistochemical study. Am J Respir Crit Care Med 2000; 162: $1577-1586$.

8 Yao W, Firth AL, Sacks RS, et al. Identification of putative endothelial progenitor cells (CD34+CD133+Flk-1+) in endarterectomized tissue of patients with chronic thromboembolic pulmonary hypertension. Am J Physiol Lung Cell Mol Physiol 2009; 296: L870-L878.

9 Lang IM, Marsh JJ, Olman MA, et al. Parallel analysis of tissue-type plasminogen activator and type 1 plasminogen activator inhibitor in plasma and endothelial cells derived from patients with chronic pulmonary thromboemboli. Circulation 1994; 90: 706-712.

10 Langer F, Schramm R, Bauer M, et al. Cytokine response to pulmonary thromboendarterectomy. Chest 2004; 126: $135-141$.

11 Kimura $\mathrm{H}$, Okada $\mathrm{O}$, Tanabe $\mathrm{N}$, et al. Plasma monocyte chemoattractant protein-1 and pulmonary vascular resistance in chronic thromboembolic pulmonary hypertension. Am J Respir Crit Care Med 2001; 164: 319-324.

12 Quarck R, Nawrot T, Meyns B, et al. C-reactive protein: a new predictor of adverse outcome in pulmonary arterial hypertension. J Am Coll Cardiol 2009; 53: 1211-1218.

13 Zabini D, Nagaraj C, Stacher E, et al. Angiostatic factors in the pulmonary endarterectomy material from chronic thromboembolic pulmonary hypertension patients cause endothelial dysfunction. PLoS One 2012; 7: e43793.

14 Mayer E, Klepetko W. Techniques and outcomes of pulmonary endarterectomy for chronic thromboembolic pulmonary hypertension. Proc Am Thorac Soc 2006; 3: 589-593.

15 Soon E, Holmes AM, Treacy CM, et al. Elevated levels of inflammatory cytokines predict survival in idiopathic and familial pulmonary arterial hypertension. Circulation 2010; 122: 920-927.

16 Humbert M, Monti G, Brenot F, et al. Increased interleukin-1 and interleukin-6 serum concentrations in severe primary pulmonary hypertension. Am J Respir Crit Care Med 1995; 151: 1628-1631.

17 Ogawa A, Firth AL, Yao W, et al. Inhibition of mTOR attenuates store-operated $\mathrm{Ca}^{2+}$ entry in cells from endarterectomized tissues of patients with chronic thromboembolic pulmonary hypertension. Am J Physiol Lung Cell Mol Physiol 2009; 297: L666-L676.

18 Bernard J, Yi ES. Pulmonary thromboendarterectomy: a clinicopathologic study of 200 consecutive pulmonary thromboendarterectomy cases in one institution. Hum Pathol 2007; 38: 871-877. 
19 Lang IM, Pesavento R, Bonderman D, et al. Risk factors and basic mechanisms of chronic thromboembolic pulmonary hypertension: a current understanding. Eur Respir J 2013; 41: 462-468.

20 Bonderman D, Wilkens H, Wakounig S, et al. Risk factors for chronic thromboembolic pulmonary hypertension. Eur Respir J 2009; 33: 325-331.

21 Wynants M, Quarck R, Ronisz A, et al. Effects of C-reactive protein on human pulmonary vascular cells in chronic thromboembolic pulmonary hypertension. Eur Respir J 2012; 40: 886-894.

22 von Haehling S, von Bardeleben RS, Kramm T, et al. Inflammation in right ventricular dysfunction due to thromboembolic pulmonary hypertension. Int J Cardiol 2010; 144: 206-211.

23 Fartoukh M, Emilie D, Le GC, et al. Chemokine macrophage inflammatory protein- $1 \alpha$ mRNA expression in lung biopsy specimens of primary pulmonary hypertension. Chest 1998; 114: Suppl. 1, 50S-51S.

24 Dorfmuller P, Zarka V, Durand-Gasselin I, et al. Chemokine RANTES in severe pulmonary arterial hypertension. Am J Respir Crit Care Med 2002; 165: 534-539.

25 Heresi GA, Aytekin M, Newman J, et al. CXC-chemokine ligand 10 in idiopathic pulmonary arterial hypertension: marker of improved survival. Lung 2010; 188: 191-197.

26 Hoh BL, Hosaka K, Downes DP, et al. Monocyte chemotactic protein-1 promotes inflammatory vascular repair of murine carotid aneurysms via a macrophage inflammatory protein- $1 \alpha$ and macrophage inflammatory protein-2dependent pathway. Circulation 2011; 124: 2243-2252.

27 Amsellem V, Gary-Bobo G, Marcos E, et al. Telomere dysfunction causes sustained inflammation in chronic obstructive pulmonary disease. Am J Respir Crit Care Med 2011; 184: 1358-1366.

28 Tatewaki H, Egashira K, Kimura S, et al. Blockade of monocyte chemoattractant protein-1 by adenoviral gene transfer inhibits experimental vein graft neointimal formation. J Vasc Surg 2007; 45: 1236-1243.

29 Saiura A, Sata M, Hiasa K, et al. Antimonocyte chemoattractant protein-1 gene therapy attenuates graft vasculopathy. Arterioscler Thromb Vasc Biol 2004; 24: 1886-1890.

30 Gharaee-Kermani M, Denholm EM, Phan SH. Costimulation of fibroblast collagen and transforming growth factor $\beta 1$ gene expression by monocyte chemoattractant protein-1 via specific receptors. J Biol Chem 1996; 271: $17779-17784$. 